\title{
Etude rétrospective du succès implantaire et prothétique à 15 ans dans un cabinet d'omnipratique. \\ Desmyttere $\mathbf{M}^{1}$, Laures $\mathrm{M}^{1}$, Fauroux $\mathrm{MA}^{1}$, Malthiéry $\mathrm{E}^{1}$, Torres $\mathrm{JH}^{1}$
}

1. Service d'Odontologie de Montpellier - Université Montpellier I : EA4203 - France

\section{Introduction}

Depuis les débuts de l'implantologie et la définition du principe d'ostéointégration par le professeur Brånemark, les implants dentaires et les techniques chirurgicales ont évolué, pour devenir aujourd'hui des outils fiables et prédictibles pour le remplacement des dents, largement utilisés par les spécialistes, et qui font aussi partie intégrante des pratiques de nombreux omnipraticiens. II reste difficile d'estimer le taux de survie des implants posés dans les cabinets d'omnipratique. La plupart des études sont en effet réalisées dans des milieux académiques. Les études menées uniquement dans les cabinets d'omnipratique peuvent être entachées de biais méthodologiques. Et on peut toujours suspecter une étude supportée financièrement par l'industrie de rapporter des chiffres complaisants. L'objet de cette étude est de présenter le recueil de données collectées dans un cabinet d'omnipratique par une équipe hospitalo-universitaire indépendante.

\section{Matériels et méthodes}

Une étude rétrospective sur 15 ans comprenant 350 implants posés chez 135 patients a été menée au sein d'un cabinet d'omnipratique de ville, par un interne DESCO. L'objet a été de déterminer les taux de succès, de survie et d'échecs implantaires, observés à long terme ( $\geq 5$ ans), les taux et les types de complications prothétiques, ainsi que les facteurs de risques associés. Les implants posés étaient tous de la marque Biotech $\circledast$; ils ont été posés par le même praticien. Les taux de succès, survie et échec implantaires ont été définis par " the health scale for dental implants » (the international congress of oral implantologists, 2008) (1). 2 radiographies (rétroalvéolaire et/ou panoramique), post-opératoires immédiates et lors du suivi ( $\geq 5$ ans) ont permis de mesurer les pertes osseuses. Résultats : Le taux de survie implantaire global observé ( $\geq 5$ ans) était de $91.7 \%$, avec un taux de complications prothétiques de $7.1 \% .15 .1 \%$ des implants ont présenté une péri-implantite. La perte osseuse marginale moyenne était de $1 \mathrm{~mm}$. Les principaux facteurs de risque identifiés (différence significative) étaient l'âge d'implantation (>50 ans et Discussion : La plupart des résultats obtenus étaient comparables à ceux de la littérature (2), à l'exception de certaines variables étudiées qui ne sont pas ou peu analysées dans cette dernière (années d'expérience, réalisation de la prothèse par les correspondants). Conclusion : Les résultats retrouvés permettent d'approcher la réalité des cabinets libéraux, et illustrent notamment un manque de suivi des patients à long terme ainsi qu'une diminution des taux de survie lorsque la pose de l'implant et la prothèse ne sont pas réalisées par le même praticien.

maxime.desmyttere@orange.fr

\section{Références}

(1) Misch CE et al. Implant Dent. 2008;17:5-15.

(2) French D et al. Clin Oral Implants Res. 2015 Nov;26:1345-54. Communication orale autorisation de changement de catégorie à la commission scientifique et de la reherche de la SFCO

(C) The authors, published by EDP Sciences. This is an Open Access article distributed under the terms of the Creative Commons Attribution License 4.0 (http://creativecommons.org/licenses/by/4.0/) 